\title{
Age May Be the Only Variable Effecting Microhematuria Prevalance in Pelvic Organ Prolapse
}

\author{
Ayse Filiz GOKMEN KARASU1', Serdar AYDIN'1, Ilknur ADANIR', Gulsah ILHAN² Senad KALKAN², Seda ATES ${ }^{1}$ \\ Istanbul, Turkey
}

\begin{abstract}
OBJECTIVE: We aimed to estimate the prevalence of microscopic hematuria in women with pelvic organ descent and compare the results in terms of severity and accompanying urinary symptoms. We hypothesized that microhematuria incidence would be higher in advanced prolapse and accompanying urinary symptoms.
\end{abstract}

STUDY DESIGN: Women who presented to our clinic from January 2013 to July 2016 were analyzed using our electronic medical record system. Those who were classified in the system under the "N81: female genital prolapse" code were included. Records were checked to certify that samples were acquired properly. Urogynecological examinations were performed by specialist gynecologists and documented according to the international continence society classification system. After ruling out urinary tract infections urinalysis was performed by an integrated system composed of an urine strip analyzer and a sediment autoanalyzer.

RESULTS: Gravidity, parity, menopause onset, smoking status and presence of systemic disease were similar for both groups. Women in the hematuria group were older $(p<0.001)$. Hematuria prevalance was greater ininternational continence society Stage 3-4 anterior prolapse and total prolapse $(p=0.03) \mathrm{com}$ pared to early stage prolapse. However after logistic regression analysis; age was the only factor left in association with hematuria.

CONCLUSIONS: Women who were older and with advanced stage prolapse $(\geq$ international continence society stage 3) were more likely to have microscopic hematuria. Urine tests are requested routinely in the work-up of urogynecological patients. As the prevalance is microhematuria is high in this population; we believe that evidence based algorithms should be set as guidelines when hematuria is encountered in patients with organ prolapse.

Keywords: Microscopic hematuria, Pelvic organ prolapse, Urinalysis

Gynecol Obstet Reprod Med 2018;24(3):143-146

${ }^{1}$ Bezmialem Vakif University Faculty of Medicine Department of Obstetrics and Gynecology, Istanbul

${ }^{2}$ University of Health Sciences Suleymaniye Research and Training Hospital Department of Obstetrics and Gynecology, Istanbul

${ }^{3}$ Bezmialem Vakif University Faculty of Medicine Department of Urology, Istanbul

Address of Correspondence: Ayse Filiz Gokmen Karasu Adnan Menderes Bulvarl Vatan St. 34093 Fatih, Istanbul, Turkey afgokmen@gmail.com

Submitted for Publication: $\quad 05.11 .2017$

Accepted for Publication: $\quad 22.12 .2017$

.:This article was presented as an abstract at the $10^{\text {th }}$ European Urogynecological Association (EUGA) Congress at Barcelona, Spain

\begin{tabular}{|c|c|}
\hline & Access this article online \\
\hline $\begin{array}{c}\text { Quick Response Code: } \\
\text { Q }\end{array}$ & $\begin{array}{c}\text { Website: www.gorm.com.tr } \\
\text { info@gorm.com.tr }\end{array}$ \\
\cline { 2 - 3 } & DOI:10.21613/GORM.2017.748 \\
\hline
\end{tabular}

How to cite this article: Gokmen Karasu AF. Aydin S. Adanir I. Ilhan G. Kalkan S. Ates S. Age May Be the Only Variable Effecting Microhematuria Prevalance in Pelvic Organ Prolapse. Gynecol Obstet Reprod Med 2018;24(3):143-146

\section{Introduction}

Microhematuria is defined as the presence of three or more red blood cells per high- power field (hpf) on a single urine sample with concurrent negative urine culture (1-4). The fastest and most convenient method of detection is via dipsticks. Despite being highly specific, dipstick testing may result in false positive results as a reaction to alkaline urine, presence of semen, myoglobinuria or contamination with chemical agents used to clean the vulva. Following centrifugation, direct observation and counting of red blood cells under a microscope is the gold standard for ascertainment of hematuria. Proper collection of urine can exclude most of the plausible causes; such as avoiding analysis following recent vaginal douching, coitus, exercise, pelvic examination or during menstruation.

Increased prevalence of microscopic hematuria among POP (pelvic organ prolapse) patients has been disputed $(5,6)$. POP frequently results in anatomical distortion of the neighbouring organs and loss of normal function. Commonly bladder involvement manifests as urinary symptoms which may accompany or precede POP symptoms (7). 
After initial history evaluation; a urine sample is a first-line fundemental test to be ordered for the evaluation of the urogynecology patient. Urinary tract infections should be ruled out as well as the presence of protein; which may indicate nephrologic disease. Also red cell casts can be noticed during microscopy which again warrants an investigation of nephrologic pathology before additional invasive tests are ordered (1).

Our objective was to estimate the prevalence and degree of microscopic hematuria in patients with pelvic organ descent. Additionally we aimed to compare the results in terms of pelvic compartment and accompanying urinary symptoms. We hypothesized that microhematuria prevalence would be higher in advanced prolapse and accompanying urinary symptoms.

\section{Material and Method}

The study was conducted at Bezmialem Vakif University Hospital, Istanbul. Instutional review board and administrative approval was obtained. This study was a retrospective analysis of patients who presented to our gynecology clinic from January 2013 to July 2016. Using our electronic medical record system patients older than 18 years old who were classified in the system under the "N81: female genital prolapse" code (International Classification of Diseases, $10^{\text {th }}$ revision) were included. Records were checked to certify proper urine sample acquisition. Samples obtained that were associated with macroscopic hematuria, menstrual discharge, pregnancy and puerperium, genitourinary tract infection, established nephrologic or urinary tract disease and recent urogynecological procedure within the past 3 months were excluded. Medical history including age, parity, smoking, menopausal status, comorbid illnesses, treatment with anticoagulants, previous gynecological and urological procedures were obtained. Physical examinations were performed by specialist gynecologists. Organ prolapse was documented by utilizing the International Continence Society (ICS) stage classification system (8). Urine samples were obtained from freshly voided mid-stream clean catch urine and sent to the same laboratory. Urinalysis was performed by Dirui Systems Automatic Urinalysis Workstation FUS-200 / H-800 Plus (Dirui Industrial Co. Changchun, China). This is an integrated system composed of an urine strip analyzer (H800 Plus) and a sediment autoanalyzer (FUS-200) which takes and stores microscopic images. Microscopic hematuria was defined as the presence of three or more red blood cells per hpf in the sediment analysis.

We analyzed the data in two major groups as regards to the presence of hematuria. All statistical analyses were performed by SPSS (Statistical Package for the Social Sciences) version 21.0 (IBM Corp, Armonk, NY, USA) statistical software. Descriptive statistics were presented as mean and standard deviations for continous variables. For categorical variables, frequency and percentage were given. Distribution of data was assessed with histogram analysis and Kruskal-Wallis test. Student's t test and Mann Whitney U test were used for the comparision of continous variables; $\chi^{2}$ and Fisher's exact tests were performed to compare categorical variables. A p value of $<0.05$ was considered significant for all tests. Multivariable logistic regression models were developed to predict the probability of microscopic hematuria.

\section{Results}

Electronic records of 843 POP patients were inspected and 506 women who met the study inclusion criteria were eligible for final analysis. Demographic data is presented in table 1. Of the 506 subjects, 114 (22.5\%) were found to have microscopic hematuria. Almost all the patients $(\mathrm{n}=111)$ had low grade

Table 1: Comparision of patient characteristics

\begin{tabular}{|c|c|c|c|}
\hline & $\mathrm{MHU}(\mathrm{n}=114)$ & Normal $(n=392)$ & $p$ \\
\hline Age $\left(\right.$ years) ${ }^{*}$ & $57.9 \pm 11.9$ & $53.7 \pm 10.4$ & $<0.0001$ \\
\hline Gravidity* & $4.2 \pm 1.9$ & $4.1 \pm 2.1$ & 0.6 \\
\hline Parity* & $3.8 \pm 1.8$ & $3.7 \pm 1.9$ & 0.8 \\
\hline Smoking, $\mathrm{n}(\%)^{*}$ & $6(5.3)$ & $9(2.3)$ & 0.1 \\
\hline Menopause, $\mathrm{n}(\%)^{\star * *}$ & $81(71.1)$ & $265(67.6)$ & 0.5 \\
\hline Blood thinner, $\mathrm{n}(\%)^{\star * *}$ & $11(9.6)$ & $20(5.1)$ & 0.2 \\
\hline Salicilic acid & $8(7)$ & $17(4.3)$ & \\
\hline Warfarin & $2(1.8)$ & $2(0.5)$ & \\
\hline Clopidrogel & $1(0,9)$ & $1(0,5)$ & \\
\hline Previous Hysterectomy, $\mathrm{n}(\%)^{\star * *}$ & $6(5.3)$ & $28(7.1)$ & 0.5 \\
\hline Previous Prolapse Surgery, $\mathrm{n}(\%)^{* *}$ & $2(1.8)$ & $2(0.5)$ & 0.2 \\
\hline Previous Incontinence Surgery, n (\%) & $2(1.8)$ & $5(1.3)$ & 0.7 \\
\hline Comorbidity, $\mathrm{n}(\%)^{* * *}$ & $57(50)$ & $189(48.2)$ & 0.7 \\
\hline Hypertension, n (\%) & $38(33.3)$ & $124(31.6)$ & 0.7 \\
\hline Diabetes, $\mathrm{n}(\%)^{* * *}$ & $18(15.8)$ & $54(13.8)$ & 0.6 \\
\hline Asthma, $\mathrm{n}(\%)^{\mathrm{c} * * *}$ & $8(7)$ & $18(4.6)$ & 0.3 \\
\hline Cystocele, $\mathrm{n}(\%)^{\star * *}$ (Any degree) & $108(94.7)$ & $379(96.7)$ & 0.3 \\
\hline$\geq$ Stage 3 anterior vaginal wall prolapse $n(\%)^{c}$ & $31(27.2)$ & $71(18.1)$ & 0.03 \\
\hline
\end{tabular}

MHU: Microscopic hematuria. Data are shown as mean+SD or $n(\%),{ }^{*}:$ Student's $t$ test, ${ }^{* *}$ :Fisher exact test, ${ }^{* * *}$ :chi-squared test. 
hematuria, defined as less than $25 \mathrm{RBC} / \mathrm{hpf}$ (9). One patient had intermediate hematuria $(25-50 \mathrm{RBC} / \mathrm{hpf})$ and two others had high grade hematuria ( $>50 \mathrm{RBC} / \mathrm{hpf}$ ). No renal pathology and no malignancy was diagnosed in our patients. Gravidity, parity, menopause onset, smoking status, presence of systemic disease were similar for both groups. History of hysterectomy, prolapse surgery and anti-incontinence surgery did not differ. Age was the only variable demographic as women in the hematuria group were older $(p<0.001)$. Thirty-one patients were on anticoagulant medication; 25 individuals were treated with asprin, 4 with warfarin and 2 with clopidogrel. Anticoagulants were not associated with increased hematuria $(p=0.07)$.

Accompanying chief urinary symptoms are given in table 2. More than half of the women $n=261(52 \%)$ with pelvic organ prolapse addressed at least one urinary complaint. The most prevailing symptom was stress urinary incontinence and was reported in 117 (44.8\%) of the sypmtomatic women. Hematuria prevalence was not different in women with urinary symptoms and no urinary symptoms $(p=0.9)$. Pelvic organ prolapse was also analyzed regarding the pelvic compartments and association with hematuria. Hematuria incidence was greater in ICS Stage 3-4 anterior prolapse $(p=0.03)$.

A logistic regression analysis was performed for the two factors reaching statistical significance (Table 3). Conclusively patient age was the only variable predicting hematuria in our cohort $(\mathrm{OR}=1.03)(\mathrm{CI}=1.01-1.06)$.

\section{Discussion}

Our aim in this study was to evaluate the prevalance of microscopic hematuria and urinary symptoms associated with pelvic organ prolapse. We hypothesised that with increasing age and increasing stage prolapse the prevalance of microhematuria would be higher. Also we hypothesised that irritative symptoms would also be higher in patients with microhemauria. The prevalence of hematuria in the general population varies according to sex, age and demographic characteristics of the population studied, as well as the chosen screening method (10-12). Our results demonstrated that the prevalence of hematuria was $22.5 \%$ in our study population. When taken into account microhematuria frequency in a subgroup of female patients with POP; researchers from Turkey have observed $10 \%$ prevalence (6). In comparison; a study from the U.S (5) reported a more similar rate to ours;20.1\%. The aforementioned study proposed that parity, smoking history and vaginal estrogen use displayed an increased association with hematuria. We did not find a significant association between parity or smoking history and microhematuria. Data regarding vaginal estrogen use was not available. In our study women who were older and whom were with advanced stage prolapse ( $\geq$ ICS stage 3 ) were statistically more likely to have microscopic hematuria. However after logistic regression analysis; age was the only factor left in association with hematuria. Some studies up to date have reported increased prevalence of microscopic hematuria among older women (13-15), whereas other reports have found no significant association with age (16). With disease progression the prolapsed vaginal part extends beyond the intraoitus rendering the vaginal mucosa more and more susceptible to abrasive trauma hence the increased hematuria prevalence in patients with advanced POP ( $\geq$ ICS stage 3 ) is comprehensible.

Women with pelvic organ prolapse often report difficulties with urination. Pelvic comorbidities such as vulvovaginal atrophy and vulvodynia may also contribute to irritative urinary symptoms. According to our results hematuria prevalence was not different in women with urinary symptoms and no urinary symptoms $(p=0.9)$. Irritative symptoms on the other hand may be a harbinger of a more serious pathology therefore we referred all women with explicit urinary symptoms and established hematuria to the urology department for further workup. When irritative voiding symptoms and hematuria are observed together; cystoscopy is recommended after risk stratification. Some investigators in female pelvic medicine advocate that the epidemiology of urinary tract malignancies is different in women and exhaustive diagnostic procedures should not be performed immedately (17). Others argue that although men are diagnosed with more bladder cancer; women present

Table 2: Microhematuria prevalance with acompanying urinary symptoms

\begin{tabular}{lccc}
\hline & MHU & Normal & $p$ \\
\hline Stres urinary incontinence & 28 & 89 & 0.08 \\
Urge urinary incontinence & 25 & 98 & 0.5 \\
Dysuria & 4 & 7 & 0.2 \\
Presence of any urinary symptom & 57 & 194 & 0.9 \\
\hline
\end{tabular}

MHU: Microscopic hematuria

Table 3: Multivariable logistic regression analysis of risk factors for the development of microscopic hematuria

\begin{tabular}{lccc}
\hline Variable & Adjusted Odds ratio & Cl & Significance \\
\hline Age & 1.03 & $1.01-1.06$ & 0.001 \\
$\geq$ Stage 3 anterior vaginal wall prolapse & 0.76 & $0.45-1.28$ & 0.3 \\
\hline
\end{tabular}

Bolding indicates statistical significance. Cl: confidence interval 
with advanced stage malignancy and have worse survival rates (18). There are studies challenging this notion. A study published in 2006 found the rate of bladder malignancy to be $0.4 \%$ in women (19). Another study comprising of 564 women with hematuria and voiding symptoms reported only one case of low-grade bladder cancer and concluded that cystoscopic evaluation is not predictive for malignancy (20).

The value of our findings in this sudy is that the rate of hematuria did not differ for premenopausal and postmenopausal women whilst increasing age was predictive of hematuria. Our interpretation of this finding is based on the fact that genitourinary atrophy status is not the same when comparing a woman in her early years of menopause and a senior menopausal patient. Additionally irritative voiding symptoms were not associated with a higher microhematuria prevalance.

The major limiation of our study was that it was conducted in a retrospective manner. Others can be listed as the lack of data regarding local estrogen treatment and objective laboratory markers of vaginal atrophy. Strenghts of our study include the considerable size of our cohort, and the proper procurement of urine for analysis.

\section{Conclusion}

In conclusion; patients with advanced stage prolapse and whom were older had a higher prevalance of microscopic hematuria. After logistic regression analysis age was the only remaining factor left in association with microhematuria. Therefore we suggest that risk stratification should be performed when microscopic hematuria is encountered in female patients presenting with POP with or without accompanying urinary symptoms.

: Conflict of Interest: We declare that we have no potential financial and non-financial conflicts of interest.

\section{References}

1. Davis R, Jones JS, Barocas DA, Castle EP, Lang EK, Leveillee RJ, et al. Diagnosis, evaluation and followup of asymptomatic microhematuria (AMH) in adults: AUA guideline. J Urol 2012;188 (6):2473-2481.

2. Wollin T, Laroche B, Psooy K. Canadian guidelines for the management of asymptomatic microscopic hematuria in adults. Can Urol Asso J 2009;3(1):77-80.

3. Horie S, Ito S, Okada H, Kikuchi H, Narita I, Nishiyama $\mathrm{T}$, et al. Japanese guidelines of the management of hematuria. Clin Exp Nephrol 2014;18(5):679-689.

4. Nederlandse Vereniging voor Urologie: Richtlijn Hematurie 2010.

5. Pillalamarri N, Shalom D, Sanidad S, Akerman M, Lind $\mathrm{L}$, Winkler $\mathrm{H}$. The prevalence of microscopic hematuria in a cohort of women with pelvic organ prolapse. Int Urogynecol J 2015;26(1):85-90.

6. Töz E, Kurt S, Canda MT, Şahin C, Uyar I. Comparison of frequency of asymptomatic microhematuria in patients with stage 2-4 versus stage 0-1 pelvic organ prolapse. Pak J Med Sci 2015;31(3):654-657.

7. Brazell HD, O'Sullivan DM, Steinberg AC. Do patients with pelvic organ prolapse have an increased frequency of asymptomatic microscopic hematuria? Urology 2014;83 (6):1236-1238.

8. Haylen B, Ridder D, Freeman R, Swift S, Berghmans B, Lee $\mathrm{J}$, et al. An International Urogynecological Association (IUGA) / International Continence Sociey (ICS) joint report on the terminology for female pelvic flor dysfunction. Int Urogynecol J 2010;21(1):5-26.

9. Loo RK, Lieberman SF, Slezak JM, Landa HM, Mariani AJ, Nicolaisen G. Stratifying risk of urinary malignant tumors in patients with asymtomatic microscopic hematuria. Mayo Clinic Proc 2013;88(2):129-138.

10. Topham PS, Jethwa A, Watkins M, Rees Y, Feehally J. The value of urine screening in a young adult population. Fam Pract 2004;21(1):18-21.

11. Hedelin H, Jonsson K, Salomonsson K, Boman H. Screening for bladder tumours in men aged 60-70 years with a bladder tumour marker (UBC) and dipstick-detected haematuria using both white- light and fluorescence cystoscopy. Scand J Urol Nephrol 2006;40(1):26-30.

12. Bangma CH, Loeb S, Busstra M, Zhu X, El Bouazzaoui S, Refos J, et al. Outcomes of a bladder cancer screening program using home hematuria testing and molecular markers. Eur Urol 2013;64(1):41-47.

13. Rodgers M, Nixon J, Hempel S, Aho T, Kelly J, Neal D, et al. Diagnostic tests and algorithms used in the investigation of haematuria: systematic reviews and economic evaluation. Health Technol Assess 2006;10:18.

14. Kawamura T, Ohta T, Ohno Y, Wakai K, Aoki R, Tamakoshi A, et al. Significance of urinalysis for subsequent kidney and urinary tract disorders in mass screening of adults. Intern Med 1995;34(6):475-480.

15. Froom P, Gross M, Ribak J, Barzilay J, Benbassat J. The effect of age on the prevalence of asymptomatic microscopic hematuria. Am J Clin Pathol 1986;86(1):656-667.

16. Hiatt RA, Ordonez JD. Dipstick urinalysis screening, asymptomatic microhematuria, and subsequent urological cancers in a population-based sample. Cancer Epidemiol Biomarkers Prev 1994;3(5):439-443.

17. Harmanli O, Yuksel B. Asymptomatic microscopic hematuria in women requires separate guidelines. Int Urogynecol J 2013;24(2):203-206.

18. Garg T, Pinheiro LC, Atoria CL, et al. Gender disparities in hematuria evaluation and bladder cancer diagnosis: a population based analysis. J Urol 2014;192(4):1072-1077.

19. Wu JM, Williams KS, Hundley AF. Microscopic hematuria as a predictive factor for detecting bladder cancer at cystoscopy in women with irritative voiding symptoms. Am J Obstet Gynecol 2006;194(5):1423-1442

20. Sokol ER, Patel SR, Sung VW, Rardin CR, Weitzen S, Clemons JL, et al. Results of urine cytology testing and cystoscopy in women with irritative voiding symptoms. Am J Obstet Gynecol 2005;192(5):1560-1565. 